\title{
Human Centered Design como camino de exploración creativa en la enseñanza-aprendizaje del audiovisual en pregrado ${ }^{1}$
}

\author{
Human-Centered Design as a Creative Exploration Path in the Teaching-Learning of \\ Audiovisual Undergraduate Students ${ }^{2}$
}

\section{Human Centered Design como um caminho exploratório criativo no ensino-aprendizagem do audiovisual na graduação ${ }^{3}$}

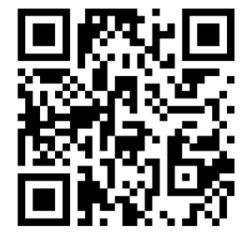

Ana María Pérez-Guerrero Universidad de La Sabana Chía-Colombia ana.perez@unisabana.edu.co iD https://orcid.org/0000-0001-9325-965X

Carlos Andrés Reyes Universidad de La Sabana Chía-Colombia carlos.reyes@unisabana.edu.co https://orcid.org/0000-0002-5858-1237

Recibido • Received • Recebido: 11 / 01 / 2020

Corregido • Revised $\cdot$ Revisado: 12 / 08 / 2021

Aceptado • Accepted • Aprovado: 09 / 10 / 2021

\footnotetext{
${ }^{1}$ Este trabajo forma parte de las indagaciones del proyecto Nuevas formas de enseñanza, basadas en Design Thinking, para favorecer procesos creativos en proyectos audiovisuales a nivel de pregrado, COD-73-2016, financiado por la Dirección de Investigación de la Universidad de La Sabana, Colombia.

2 This work is part of research project titled New forms of teaching, based on Design Thinking, to favor creative processes in audiovisual projects at the undergraduate level, COD-73-2016, funded by the Research Directorate of the University of La Sabana, Colombia.

${ }^{3}$ Este trabalho faz parte das investigações do projeto Novas formas de ensino, baseadas no Design Thinking, para favorecer processos criativos em projetos audiovisuais de graduação, COD-73-2016, financiados pela Diretoria de Pesquisa da Universidade de La Sabana, Colômbia.
} 
http://doi.org/10.15359/ree.26-1.8

http://www.una.ac.cr/educare

educare@una.ac.cr

\begin{abstract}
Resumen:
Objetivo. Determinar los beneficios del empleo del Human Centered Design en la formación audiovisual a nivel de pregrado, en el caso del alumnado de la asignatura Géneros y Formatos Audiovisuales, del tercer semestre de la carrera en Comunicación Audiovisual, en la Universidad de La Sabana (Colombia). Metodología. Este caso estudio, de enfoque cualitativo, aborda la aplicación de los principios del HCD contenidos en los pasos de Design Thinking, por parte del estudiantado en el desarrollo de creación de un cineminuto. Participan 177 estudiantes, durante el periodo comprendido desde 20182 a 2019-2. A partir de instrumentos de recolección de datos como la observación participante, las revisión documental y audiovisual, resultante de cada fase del proceso, se analizan tres categorías: la seguridad creativa, la resolución de problemas creativos y el conocimiento adquirido por el alumnado. Resultados. La aplicación de HCD mostró avances en el aprendizaje del alumnado en los aspectos estudiados, aunque plantea desafíos en el terreno del trabajo en equipo. Conclusiones. HCD resulta una forma efectiva de presentar al estudiantado un camino de exploración para la creación audiovisual, que fomenta la seguridad creativa, mejora el abordaje de problemas narrativos y técnicos, mediante la evaluación y prototipado de sus trabajos; por tanto, consolida los conocimientos adquiridos tanto en clase como en fases de empatía, (investigación).
\end{abstract}

Palabras claves: Creación audiovisual; didáctica audiovisual; educación universitaria; estrategia didáctica; diseño-centrado en personas; pensamiento de diseño.

\begin{abstract}
:
Objective. To determine the benefits of using Human-Centered Design (HCD) in audiovisual training, at the undergraduate level, in the case of students of the Audiovisual Genres and Formats subject. The students are enrolled in the third semester of the Audiovisual Communication program at the University of La Sabana (Colombia). Method. With a qualitative approach, this case study addresses the application of the HCD principles contained in the Design Thinking steps by the student body in the creation process of a cineminuto. During the period from 2018-2 to 2019-2, 177 students participate in the training. From data collection instruments such as participant observation and documentary and audiovisual reviews, resulting from each phase of the process, three categories were analyzed: creative confidence, creative problem solving, and knowledge acquired by students. Results. The application of HCD showed advances in student learning in the aspects studied, although it poses challenges in the field of teamwork. Conclusions. HCD is an effective way of presenting a path of exploration for audiovisual creation to students; it fosters creative security and improves the approach to narrative and technical problems through evaluating and prototyping their work. Therefore, it consolidates the knowledge acquired both in class and in phases of empathy (research).
\end{abstract}

Keywords: Audiovisual creation; audiovisual didactic; creativity; design thinking; teaching strategy; human-centered design; university teaching.

\title{
Resumo:
}

Objetivo. Determinar os benefícios do uso do Human Centered Design na formação audiovisual de graduação, no caso de estudantes da disciplina de Gêneros e Formatos Audiovisuais, do terceiro semestre da carreira em Comunicação Audiovisual, da Universidade de La Sabana (Colômbia). Metodologia. Este estudo de caso, com abordagem qualitativa, aborda a aplicação dos princípios de HCD contidos nas etapas do Design Thinking, pelo corpo discente no desenvolvimento da 
criação de um cineminuto. Participaram 177 estudantes, durante o período de 2018-2 a 2019-2. A partir de instrumentos de coleta de dados como observação participante, revisão documental e audiovisual, resultantes de cada fase do processo, três categorias são analisadas: segurança criativa, resolução criativa de problemas e os conhecimentos adquiridos pelos estudantes. Resultados. A implementação do HCD deixou perceber avances no aprendizado dos estudantes nos aspectos estudados, apesar de apresentar desafios na area do trabalho em equipe. Conclusão. O HCD é uma forma eficaz de apresentar aos estudantes um percurso de exploração para a criação audiovisual, que fomenta a segurança criativa, melhora a abordagem de problemas narrativos e técnicos, através da avaliação e prototipagem do seu trabalho; portanto, consolida os conhecimentos adquiridos tanto nas aulas quanto nas fases de empatia (pesquisa).

Palavras-chave: Criação audiovisual; ensino audiovisual; ensino universitário; estratégia didática; desenho centrado nas pessoas; pensamento sobre desenho.

\section{Introducción}

La enseñanza de la escritura y la realización audiovisual a nivel de pregrado entraña el reto de formar al estudiantado en conocimientos teóricos fundamentales, que se entrelazan con la aplicación técnica y con su propio talento para resolver los problemas creativos propios de cada proyecto, en las oportunidades de práctica que brinda el contenido programático; amén de favorecer en ellos cierta comprensión del proceso creativo como una vía de entendimiento de su propia sensibilidad y del dominio de algunas competencias, así como de conexión con otros. No en vano, en el resultado de su esfuerzo, -el relato-, se conjugan la expresión personal y la comunicación.

Y es este último aspecto, en el que se requiere, necesariamente, la actitud y capacidades del alumnado, el que hace más desafiante la labor docente en esta área, máxime cuando buena parte de los que ejercen en estas cátedras son profesionales en sus especialidades, pero no tienen muchas herramientas pedagógicas para animar a sus discípulos a ir más allá de la técnica. Tanto es así que algunos directores de cine, que se han dedicado, por años, a la educación superior, en este campo afirman que dirigir y escribir para la pantalla solo se pueden aprender mediante un proceso autodidacta (Mackendrick, 2004), en el que imperen la experiencia propia en rodaje y la posibilidad de análisis de ejemplos. Sin embargo, como señala Hurbis-Cherrier (2018), también el profesorado puede asumir un papel de guía en este punto, para lo que se precisa de estrategias que le permitan acompañar a sus estudiantes en su aprendizaje sin ahogar el trabajo que a este grupo compete.

Por su parte, la perspectiva de Human Centered Design (HCD) y, especialmente, su método design thinking (DT) se han destacado por brindar una sistematización del trabajo interdisciplinar orientada a la resolución innovadora y significativa de problemas, a partir de la empatía con el ser humano como factor clave en cada fase del proceso (Kramer et al., 2016), fomentando la creatividad, la solidaridad y el impulso indagatorio en las personas involucradas en la 
http://doi.org/10.15359/ree.26-1.8

http://www.una.ac.cr/educare

educare@una.ac.cr

situaciones a resolver, que participan de estos procesos. Con técnicas versátiles han logrado su aplicación exitosa en diversidad de ámbitos, entre los que se cuentan la educación, en diferentes niveles y con distintos propósitos. Por tanto, cabe preguntarse si HCD podría contribuir a que el estudiantado de audiovisual tenga una mayor comprensión del proceso creativo en este terreno, en el que se contempla el uso de los géneros y formatos audiovisuales.

El presente trabajo, de enfoque cualitativo, tiene por objeto determinar los beneficios del empleo de HCD en la formación audiovisual a nivel de pregrado, mediante el caso de estudio de la aplicación de los principios de esta perspectiva metodológica y, en concreto, de los pasos de DT, por parte del alumnado de la asignatura Géneros y Formatos Audiovisuales, del tercer semestre de la carrera en Comunicación Audiovisual, en la Universidad de La Sabana (Colombia), entre los periodos 2018-2 a 2019-2. La elección de esta perspectiva responde a la necesidad de encontrar aproximaciones educativas que sean respetuosas con la naturaleza de la creación de contenidos audiovisuales de ficción, así como con el proceso de enseñanza-aprendizaje en este campo, con la aspiración de fomentar en el alumnado un entendimiento de la mecánica narrativa audiovisual, así como el alcance de sus trabajos y de su rol creador, mediante un camino que les implique y facilite su autoconocimiento.

Para lograr este cometido, se presenta una breve panorámica de algunos modelos y trabajos basados en el empleo de HCD y DT en la educación superior, incluyendo algunas experiencias en el terreno audiovisual. Seguidamente, se exponen los conceptos fundamentales del método, que posibilitan que se plantee como una vía pedagógica en esta área y se comentan algunas de las formas en que se ha empleado en la realización de películas. A continuación, se explica el diseño de la puesta en marcha de HCD en la experiencia en la que se centra este artículo, el procesamiento de los datos obtenidos durante su realización, así como los resultados obtenidos con relación con las competencias estudiadas: seguridad creativa, resolución de problemas creativos y conocimiento del género audiovisual, para dar paso a las conclusiones extraídas del caso, en las que HCD, presenta varios beneficios al alumnado, sobre todo, en la esfera del autoconocimiento creativo.

\section{HCD y DT aplicados a la educación}

Los estudios de HCD aplicados a la educación superior son abundantes, especialmente, en el campo del diseño industrial, el desarrollo de software, en ingeniería y en la gestión (Boy, 2013; Steen, 2012), mientras los que abordan la formación de la creatividad audiovisual son poco menos que escasos. Sin embargo, es gracias a trabajos sobre DT en esta área que se amplían los tópicos y se presentan diferentes modelos. Uno de los más destacados por su vocación generalista es Evolution E6 ${ }^{2}$, de la Escuela de Diseño (Portugal), que se plantea como herramienta transversal de enseñanza en educación universitaria y formación profesional con fines diversos de aplicación.

4 
http://doi.org/10.15359/ree.26-1.8

Las bases de este proyecto se desarrollan en Research Report D-Think (Tschimmel et. al., 2015), donde se remarca el carácter evolutivo, iterativo e interactivo del proceso de creación, que involucra a personas y situaciones. La complejidad de este modelo contrasta con otros paradigmas similares, aunque mantiene un carácter polivalente, que se establece en su guía D-Think Toolkit (Tschimmel et. al, 2017).

En lo que respecta a la bibliografía sobre DT en educación universitaria, resulta numerosa, sobre todo, en áreas como la ingeniería y los temas de índole organizacional. Series como la de Springer, Understanding Innovation, editada por Meinel y Leifer, trata el tema de manera transversal, aunque es posible mencionar las ediciones de Brenner y Uebermickel (2016) que dedican partes enteras a la enseñanza. Sin embargo, en lo que se refiere a artículos se aprecia una variedad mayor de tópicos, abordando asuntos curriculares (Kramer et al., 2016) y experiencias innovadoras que demuestran las bondades de la metodología (Majithia y Burman, 2015; Steinbeck, 2011). Dentro de este último grupo, vale la pena mencionar el trabajo de Lugmayr et. al (2014), en la Universidad de Tecnología de Tampere, con el que se busca estimular al alumnado a crear nuevas ideas en la industria de los medios, aprovechando el carácter cooperativo, interdisciplinario de DT e incorporarlo a temas organizacionales y de innovación, proveyendo una guía para quien quiera implementar un curso de este tipo a nivel superior. Asimismo, cabe mencionar la iniciativa del laboratorio, Relatos del patrimonio en clave digital (Rodríguez Rojas y Mosquera, 2018), del grupo de investigación Escenarios de Narración Transmedia para la Documentación y Visibilidad del Patrimonio Cultural de la Ciudad de Bucaramanga, realizado por la Universidad Autónoma de Bucaramanga (Colombia) y la Universidad de Santander (Colombia), en el que DT es el canal de desarrollo creativo de treinta participantes orientado a difundir el patrimonio local mediante contenido transmedia.

Finalmente, en la práctica audiovisual universitaria hay que señalar algunas experiencias desarrolladas en semilleros de investigación, como, por ejemplo, el proyecto Emociones de la Universidad Autónoma de Bucaramanga y la Agencia para la Reincorporación y Normalización de Santander, en Colombia, donde se emplea DT en el desarrollo de cineminutos documentales destinados a concienciar a la ciudadanía sobre la reconciliación y la convivencia en el post conflicto del país, así como los proyectos de Sigla, en la Universidad de La Sabana, en los que se usa HCD como base para la resolución de problemas de producción y guion de ficción. Igualmente, hay que mencionar el curso Movie Design, de la Escuela de diseño de Stanford, que ofrece aprendizaje basado en proyectos a partir de DT, haciendo énfasis en el prototipado como estrategia para abordar eficaz y rápidamente los desafíos de la realización. No obstante, estos ejemplos consisten en iniciativas inéditas, que evidencian la necesidad de un mayor número de publicaciones científicas que registren las aportaciones de HCD y DT a la enseñanza de la creatividad audiovisual, entendida como aquella que tiene por objeto la resolución de problemas creativos en el marco de las diferentes fases destinadas a la realización de un contenido de este tipo, orientado a un público específico, a través de plataformas tecnológicas o medios determinados. 
http://doi.org/10.15359/ree.26-1.8

http://www.una.ac.cr/educare

educare@una.ac.cr

\section{Fundamentos de HCD como enfoque posible para la enseñanza audiovisual}

HCD constituye un marco dentro del que se cuentan métodos y principios estratégicos de creación dirigidos a resolver problemas de diseño para que productos y servicios sean significativos para su público usuario, a partir de la aplicación del conocimiento del ser humano adquirido al observar su interacción con el entorno, contemplando sus necesidades y aspiraciones concretas en los ámbitos y situaciones específicas que constituyen los desafíos a atender desde este enfoque (van der Bijl-Brower y Dorst, 2017). De ahí que el énfasis en las personas incluya también a los grupos de interés relacionados con las cuestiones a solucionar, para alcanzar mejores resultados (IDEO.org, 2015).

El origen de HCD se remonta a mediados del siglo pasado, cuando se hizo necesario considerar al sujeto usuario en las áreas de ergonomía, informática e inteligencia artificial. Sin embargo, este imperativo fue derivando en una mentalidad que trasciende el solo perfeccionamiento de productos, lo que facilita un diálogo entre estos y la cultura, entre estos y las muchas miradas que un individuo puede tener de él (Krippendorff, 2006; van der Bijl-Brower y Dorst, 2017). Surge, entonces, un modo de resolver problemas que, si bien adopta algunos rasgos del diseño, -como la iteración y el prototipado-, se abre a diversas disciplinas, para conectar mejor con las personas y sus retos en ámbitos particulares.

Así, las técnicas enmarcadas en HCD pasan por tres fases fundamentales: la inspiración, la ideación y la implementación, se basan en la empatía con la persona usuaria como clave de la investigación y definición de las cuestiones relevantes en los temas que se pretenden mejorar, y para idear las posibles soluciones; igualmente, el prototipado y la evaluación suponen un pilar del proceso, pues facilita la correcta puesta en marcha del resultado. Dentro de este marco se cuentan, entre otros, el diseño inclusivo, el diseño basado en la innovación y el DT, que ha sido quizás la metodología que más ha contribuido a la popularización de HCD, mediante un sencillo modelo propuesto por la escuela de diseño de Stanford y la consultora IDEO en las que se aplican las fases antes señaladas, en las que caben diversidad de dinámicas y técnicas.

Debido al carácter iterativo de este proceso, las fases que lo componen no dibujan una trayectoria necesariamente secuencial tras la primera evaluación, sino según las necesidades del proyecto, por ejemplo, se puede pasar de la ideación a la empatía o a la definición. Cabe destacar que las fases de HCD y su dinámica no lineal son compatibles con procesos creativos diversos que incluyen el del audiovisual.

En tal sentido, otro aspecto relevante de HCD que puede ser de utilidad en la formación en audiovisual es el tipo de asuntos que está destinado a resolver, dado que comparten una misma condición, por la que son conocidos como problemas perversos (wicked problems), término acuñado por Rittel en "Dilemmas in a General Theory of Planning" (1973), para designar a aquellas cuestiones que se producen en sistemas complejos o confusos, que muchas veces 
http://doi.org/10.15359/ree.26-1.8

están mal formuladas, pero que no requieren de una respuesta taxativa, debido a la naturaleza altamente ambigua de sus cualidades.

Queda claro, como afirma Camillus (2008), que hablar de un problema perverso no consiste en señalar un grado de dificultad, sino un tipo de realidades que se producen en sistemas complejos, en las que intervienen diferentes causas y variadas formas de resolver las cuestiones, por lo que suelen intervenir grupos de personas involucradas en la situación, aplicando sus diferentes experiencias y conocimientos, a fin de lograr las salidas más adecuadas. Esta posibilidad de participación contribuye al empoderamiento de las comunidades, fortaleciendo su seguridad creativa, en la que reside, según Kelley y Kelley (2013) el corazón de la innovación y podría decirse también, que del aprendizaje.

La seguridad creativa consiste en la confianza que una persona adquiere en sus propias habilidades para resolver problemas en un entorno incierto (Jobst et. al., 2012), trascendiendo los conocimientos profesionales que posea. Este concepto se fundamenta en la teoría para el cambio del comportamiento de Bandura (1997), denominada, autoeficacia, que alude al efecto que tiene sobre la conducta y el desempeño las creencias que cada persona tiene sobre sus propias capacidades de organizar y ejecutar las acciones requeridas para producir unos logros determinados.

La autoeficacia es una cualidad indispensable para HCD, especialmente, cuando se trata de buscar soluciones que aún no se han descifrado por completo (IDEO.org, 2015); constituye una fuerza que impulsa a proponer y probar salidas a cuestiones en disciplinas como los negocios, la adquisición de nuevo conocimiento o la producción creativa asociada al arte, por lo que resulta una aspiración óptima para la formación en audiovisual.

\section{HCD y el proceso creativo audiovisual}

HCD y, en concreto, DT han dado muestras de favorecer la creación audiovisual en algunos aspectos, principalmente en el guion, gracias a que las operaciones de escritura se relacionan con el diseño (Leverenz, 2014). No en vano, el arte narrativo pasa por empatizar (investigar), definir (premisas, temas), idear (tramar/escribir), prototipar y evaluar (reescrituras) de manera orgánica y no lineal, si bien esto mismo no resulta tan natural cuando se equipara a los pasos de DT con los de la realización audiovisual, puesto que ella supone procesos altamente especializados y tipificados (pre-producción, producción y posproducción). Sin embargo, esto no ha sido impedimento para que algunos cineastas independientes hayan apelado a DT en diversos momentos de estas fases para lograr objetivos determinados.

En el terreno del documental, por ejemplo, las dinámicas de empatía pueden favorecer el trabajo de investigación y la conexión con los personajes de los que trata una obra. Del mismo modo, en contenidos de ficción, el estudio profundo del tema a desarrollar puede contribuir 
http://doi.org/10.15359/ree.26-1.8

http://www.una.ac.cr/educare

educare@una.ac.cr

significativamente a la definición de los conflictos de una trama e incluso a la consecución de inversionistas, como ocurrió en el filme, For Here or to Go? (Humnabadkar, 2015), cuya historia aborda la complejidad de las políticas migratorias estadounidense, a través de las peripecias de un aspirante a empresario tecnológico en Silicon Valley, proveniente de India. Cinta para la que fue fundamental las experiencias de cincuenta familias inmigrantes de ese país, que mediante entrevistas pudieron nutrir el relato (D'Sousa, 2016). Asimismo, el interés primordial de DT de ofrecer soluciones significativas, se ha planteado como método posible para contribuir al mejoramiento de la exhibición y la promoción de contenidos, a partir del estudio de la experiencia de los usuarios de las salas de cine (Continuum, 2012).

Si bien el DT puede contribuir a resolver problemas concretos dentro de alguna fase de la realización, también es claro que su empleo en el marco de la creatividad audiovisual no supone la adopción de todas las dinámicas recomendadas en los manuales publicados del método, sobre todo, si se tiene en cuenta su utilidad frente a mecánicas ya institucionalizadas en el sector y que dan frutos similares. En este sentido, cobran especial relevancia acciones que el cine ha cultivado por años para comprender a su audiencia o para tener una mayor optimización económica y de recursos logísticos, como, por ejemplo, los grupos focales, tan socorridos desde los años cuarenta, o las distintas técnicas de pre-visualización establecidas para cubrir los imperativos de producción de diferentes tipos de contenidos, como el empleo de storyboards y animáticas, que permiten ver la obra en proceso y evaluarla, como ocurre en animación, en publicidad o en producciones del género de acción.

\section{Diseño de la implementación de HCD para la enseñanza audiovisual}

Teniendo en cuenta el objetivo de este estudio, se opta por indagar qué beneficios ofrece HCD al estudiantado de la carrera audiovisual en aspectos que este método implica, como la seguridad creativa, la manera de aproximarse a problemas perversos y en el conocimiento de los temas de una asignatura específica, procurando que sea el alumnado mismo quien se cuestione el desarrollo de su proceso creativo y encuentre las soluciones a los retos estéticos, narrativos y técnicos del proyecto audiovisual que se le asigne ${ }^{4}$. Por tanto, se aprovecha el vínculo intrínseco entre HCD con DT para perfilar un proceso en el que se vivan las fases propuestas por este último, debido a su sencilla estructuración.

Para el seguimiento y análisis de todo el proceso del estudiantado se recolectan datos de carácter documental escrito y audiovisual que atestiguan su reflexión sobre la creación audiovisual y su entendimiento de la narrativa genérica empleada en su proyecto de clase, el

\footnotetext{
${ }^{4}$ Los retos que proponen los proyectos audiovisuales son únicos de cada proyecto, ya que estos dependen de la historia que se quiera relatar. En este caso, eso incluye al tratamiento que se haga desde el género.
} 
http://doi.org/10.15359/ree.26-1.8

cual consiste en un cineminuto; categoría supragénerica que, por su corta duración, facilita que se pueda prototipar y evaluar, al menos dos veces, durante el semestre.

En concreto, se hace acopio de las entregas realizadas por el estudiantado durante el proceso, las cuales se dividen en documentos escritos de creación (sinopsis, guion y propuesta de producción) y documentos escritos de evaluación, en los que el alumnado evalúa el proceso del proyecto (autoevaluación y decisiones creativas tomadas tras la realización de grupos focales y el documento final con el que se cierra la reflexión sobre su aprendizaje). Asimismo, con el fin de determinar perspectivas individuales del alumnado, se estudia la respuesta a una pregunta incluida en cada uno de los tres parciales que se presentan en el semestre, en la que se solicitan cinco conclusiones referentes al proceso. En cuanto a los documentos audiovisuales, se toman en cuenta el resultado del rodaje de prueba (prototipo) y el cortometraje final de clase.

Cabe destacar que el profesorado asume la observación participante del proceso, especialmente en los escenarios en los que se desarrollan actividades de autoevaluación y coevaluación por parte del alumnado, así como en las asesorías destinadas al seguimiento de cada equipo. Cada fase es valorada mediante rúbrica y realimentada de manera independiente por el profesorado y el monitor de la materia (alumnado notable de semestres más avanzados), a fin de tener puntos de vista distintos en el análisis del desempeño del estudiantado.

\section{HCD en la clase de Géneros y Formatos, la puesta en marcha}

La implementación del HCD se realiza desde el semestre 2018-2 hasta 2019-2, en el marco de la asignatura Géneros y Formatos Audiovisuales del tercer semestre de la carrera Comunicación Audiovisual y Multimedios de la Universidad de La Sabana. En total, participan 177 estudiantes y se realizan 30 proyectos.

Se elige esta materia, debido a su naturaleza teórico-práctica, cuyo objetivo, según el programa de la materia, "es el estudio y aplicación de los fundamentos de los géneros y formatos audiovisuales, atendiendo a sus componentes narrativos y formales" (Universidad de La Sabana, Facultad de comunicación, 2018, p. 2), debiéndose concretar en la creación de una pieza audiovisual de sesenta segundos de duración en el que se aprecien, al menos, los elementos básicos del género canónico asignado por sorteo (arquetipo, trama, iconología...). El establecimiento de esta medida de evaluación responde al nivel del grupo, pues en este momento de la carrera el alumnado tiene un conocimiento básico del lenguaje audiovisual y un desarrollo casi nulo en competencias relacionadas con la escritura para la pantalla, ya que aún no han cursado la asignatura de guion.

Por otra parte, los géneros audiovisuales se definen como una realidad polisémica, que atañe al proceso integro de los contenidos de esta índole. Se plantean como perspectivas desde las que la narración explora la condición humana, suponiendo un tratamiento retórico 
http://doi.org/10.15359/ree.26-1.8

http://www.una.ac.cr/educare

educare@una.ac.cr

particular de las historias, que incide de manera directa en la elección de una película o programa televisivo por parte de la audiencia, al presentárseles como promesa emocional y, por tanto, se convierte en punto de referencia para su distribución y promoción. De este modo, la materia facilita que el estudiantado aplique HCD a buena parte del proceso creativo: guion y desarrollo, preproducción, producción, posproducción y distribución.

El diseño de la puesta en marcha de esta perspectiva metodológica resulta, en parte, de su aplicación en el semillero de guion y realización audiovisual a cargo del equipo investigador del proyecto que cobija esta experiencia, el cual ha ofrecido resultados en relación con la eficacia de HCD y DT en el fomento de la creatividad, la cocreación, la gestión de la producción y la distribución audiovisual (Forero Serna, 2018; Pérez Guerrero y Reyes López, 2016; Rodríguez Martín, 2018). Sin embargo, el traslado a un escenario curricular como el aula supone atender al contenido programático desplegado en dieciséis semanas y a un mayor número de participantes. En consecuencia, se estima pertinente que el empleo de HCD se realice a través del trabajo independiente, destinado al proyecto final de la clase: un cineminuto orientado a todo público y un documento en el que se da cuenta de su proceso creativo.

La aplicación de HCD en el desarrollo del proyecto final permite su convivencia con las clases y otras asignaciones; facilita que el estudiantado siga la metodología de HCD de manera autónoma y al profesorado le brinda la posibilidad de valorar el cumplimiento de las fases de ese método por parte de los equipos, así como el influjo de este en su aprendizaje, mediante un proceso que genera evidencias paso a paso en los aspectos clave del HCD, como los que se persigue indagar en el presente trabajo.

Cada aula cuenta con hasta treinta y dos estudiantes que se distribuyen en equipos de cinco o seis personas, cuyos integrantes son elegidos por el mismo grupo, entre los que se reparten los cargos para asumir la dirección, producción, dirección de arte, dirección de fotografía, edición y sonido.

El proceso experimentado por el estudiantado se describe en el marco del HCD y DT de la siguiente manera:

Inspiración (empatía): etapa de investigación, que se concreta en la revisión documental sobre el género asignado más allá de los materiales provistos por la clase y la indagación necesaria para la creación del relato, así como la realización de grupos focales en los que se atiende el punto de vista de una audiencia ajena al mundo audiovisual.

Ideación (definición e ideación): durante el proceso creativo se busca que en cada entrega el alumnado identifique los desafíos que le plantea la producción y que diseñe (idee) una forma de resolverlos, justificando las medidas que haya decidido. Asimismo, en las actividades de coevaluación, debe determinar cómo contribuir a la mejora de los proyectos de los compañeros y compañeras. 
http://doi.org/10.15359/ree.26-1.8

Implementación (prototipado y evaluación): el prototipado se materializa en las distintas reescrituras de un guion o en las diferentes formas de pre-visualización de la historia (Millard, 2014), de modo que cada entrega y su evaluación por parte del estudiantado, sus pares, el profesor o profesora, quien monitorea y la audiencia conforman estas fases de la iteración esenciales en DT, en procura de inspiración y perfeccionamiento del proyecto.

\section{La estrategia en detalle}

Desde la primera semana de clase, en cada uno de los semestres en los que se realiza el estudio, se explican sus objetivos, beneficios, limitaciones y procesos al alumnado, con el fin de que manifiesten su deseo o no de participar en el proyecto, mediante consentimiento informado oral, en el que también se obtiene su autorización para la toma de fotografía de sus exámenes sin revelar su identidad. Este paso es necesario, pues, aun cuando este trabajo no compromete la integridad física del estudiantado -ya que solo se analizan sus productos de clases y sus impresiones del proceso-, se entiende la presencia de un riesgo mínimo al tratarse de población subordinada y, por tanto, se da garantías de confidencialidad, autonomía y libertad de participación, subrayando que sea cual sea la decisión del alumnado al respecto, incluso si desean retirarse durante el desarrollo de la investigación, no afectará en modo alguno sus resultados en la asignatura, ni el trato que el personal docente les dispense. Además, en caso de que el alumnado perciba alguna inconsistencia de cualquier orden con lo expuesto en el consentimiento, puede conversarlo con el personal docente a cargo o con las instancias superiores de la Facultad, encargadas de todo lo que concierne a los actores involucrados en el proyecto.

Por otra parte, se entregan cinco instructivos con sus rúbricas correspondientes: en el primero se describen los requerimientos generales del proceso, en el que se indican las fechas importantes y las características del proyecto final, y cuatro más, centrados en las entregas específicas (una por mes), correspondientes a cada fase del desarrollo del proyecto:

1. Instructivo general (empatía): se dan las claves del trabajo y se advierte de la necesidad de una lista de utilizables como punto de partida creativo, que consiste en una relación de objetos y lugares de los que el estudiantado disponga para la realización del proyecto, con la finalidad de fomentar la creatividad y lograr la viabilidad económica de las piezas.

2. Sinopsis, lista de utilizables e identificación de rasgos genéricos en el relato (definición, ideación, prototipado): texto explicativo de los aspectos prototípicos del género presentes en la propuesta, así como la lista de utilizables del equipo y los responsables de los objetos, que se deben apreciar en la sinopsis. La historia puede tener un autor o autora, pero todo el grupo debe contribuir a su perfeccionamiento. 
http://doi.org/10.15359/ree.26-1.8

http://www.una.ac.cr/educare

educare@una.ac.cr

3. Guion + storyboard + primera parte del documento final (ideación, prototipado): documento en el que se incluyen las propuestas de cada uno de los departamentos sobre cómo ven la historia y cómo contribuirán a la visión del director o directora.

4. Animática + grupo focal (prototipado, evaluación, empatía, definición): la animática del corto se prueba en grupos focales (heteroevaluación) integrados por veinte personas ajenas al audiovisual, con el fin de verificar la comprensión de la historia, la comunicación del género y lo más importante: definir las decisiones creativas que el estudiantado adopta para mejorar el producto en un primer rodaje del corto (autoevaluación), las cuales se exponen en un documento que entrega al profesorado a cargo y a la persona monitora. Luego, en clase, se presenta la pieza al grupo del aula, quienes, tras el visionado del cineminuto y la escucha de los cambios que harán al cortometraje por parte de su director o directora, comentan dichas decisiones y hacen algunas aportaciones (coevaluación). Por último, el profesor o profesora y el encargado de monitor, si está ese día en el aula, tienen la palabra.

5. Rodaje de prueba + grupo focal + conclusiones (prototipado-evaluación- empatíadefinición-ideación): una vez aplicadas las decisiones de mejora en un primer rodaje, se repite el anterior proceso de valoración del cineminuto, del que se extraen nuevas decisiones creativas y nuevas conclusiones, que se comentan en clase.

6. Entrega final, cineminuto + documento (evaluación): se envía al profesor o profesora $y$ al monitor o monitora un documento en el que se plasma el proceso, se explican los cambios, el aprendizaje, la estrategia de distribución del corto, así como el enlace al cineminuto definitivo.

7. Muestra final: encuentro que consiste en la proyección del corto del alumnado y sus personas invitadas, en la que pueden comprobar el avance o no de proyectos al término del semestre.

\section{Procesamiento de la información}

La información documental y audiovisual recolectada se procesa de manera manual y mediante procesador de texto. Se codifican los datos concernientes al alumnado, con el fin de salvaguardar su identidad, y se clasifican por cohortes. Se analizan los documentos escritos y audiovisuales del alumnado, así como las observaciones del personal docente a la luz de indicadores que permitan comprobar la evolución grupal e individual del estudiantado en las unidades de análisis, que se derivan de aspectos relacionados con la implementación del método, como la seguridad creativa y la resolución de problemas perversos. Además, dada la incidencia de estos rasgos previstos en el desempeño académico del alumnado, se aborda el conocimiento adquirido por este, en relación con el género audiovisual (ver tabla 1). 
http://doi.org/10.15359/ree.26-1.8

Tabla 1: Procesamiento de la información

\begin{tabular}{lll}
\hline \multicolumn{1}{c}{ U. de análisis } & \multicolumn{1}{c}{ Fuente informativa } & \multicolumn{1}{c}{ Indicadores } \\
\hline Seguridad creativa & - Documentos creativos & - Superación de retos individuales \\
& - Documentos de evaluación entregados & - Certeza de lo aprendido \\
& por el estudiantado & - Cambios en la actitud \\
& - Pregunta final de parciales & - Entendimiento del rol dentro del \\
& - Observación del profesorado & equipo \\
\hline Solución a los problemas & - Documentos creativos & - Superación del desafío principal del \\
creativos del proyecto & - Documentos de evaluación entregados & proyecto \\
& por el estudiantado & - Criterios para la toma de decisiones \\
& - Observación contexto & - Investigación \\
\hline Conocimiento sobre el & - Documentos de evaluación & - Reflexión sobre el género en las \\
género audiovisual & - Documentos de creación & propuestas creativas \\
& - Observación del profesorado & - Aplicación de convenciones del género \\
\hline
\end{tabular}

Nota: Elaboración con datos propios.

La unidad de análisis que aborda la seguridad creativa estudia las creencias de las personas participantes sobre el desempeño creativo y los cambios de actitud que estas produzcan en ellas. Por tanto, los indicadores que describen esta unidad son de carácter individual y se centran en cómo el logro de los desafíos de producción de un proyecto consigue un cambio de actitud, que se relaciona con la certeza de lo que se sabe o aprende y con una mayor comprensión del papel de cada cargo que se ocupa en el equipo de producción.

Por su parte, la unidad de análisis concerniente a la solución de problemas creativos se centra en la reflexión e investigación que llevan a un equipo al logro principal de su proyecto. En tal sentido, mediante sus documentos creativos y de evaluación se busca determinar el abordaje que hace el grupo de su proyecto: si se emplea el recurso a una investigación mediante recursos distinto de clase, si los criterios para la solución de los problemas están justificados, desde lo técnico o narrativo, y si se consigue vencer el reto propuesto por el cortometraje.

En cuanto al conocimiento del alumnado sobre el género audiovisual, se analiza la aplicación de las convenciones de este al cineminuto, por parte de los equipos y la justificación que ellos manifiestan sobre sus decisiones creativas. Este aspecto es crucial en dos sentidos: en el primero, se considera necesario comprobar que la adquisición de conocimiento está relacionada con la seguridad creativa y el fortalecimiento en la capacidad de resolver problemas de una manera adecuada; y en el segundo, porque es preciso ver el influjo de la metodología en la comprensión del género narrativo audiovisual en el alumnado, como algo más profundo y orgánico en las historias que la simple aplicación de convenciones. 
http://doi.org/10.15359/ree.26-1.8

http://www.una.ac.cr/educare

educare@una.ac.cr

En cuanto al análisis de cada unidad se determina, equipo por equipo, el cumplimiento pleno, parcial o no de los indicadores, mediante codificación para cada caso. Se buscan repeticiones y diferencias, y se exploran las causas, de manera especial en los casos singulares, como puede ser, por ejemplo, cumplimientos parciales de indicadores o resultados atípicos, mediante nueva revisión de los documentos y las observaciones. Asimismo, se retiran los documentos incompletos. Finalmente, se comparan las unidades de análisis entre sí para verificar su interrelación.

\section{HDC, un camino de descubrimiento en dos direcciones}

La observación y seguimiento de la implementación de HCD en la clase de Géneros y Formatos arroja resultados muy positivos en los trabajos del estudiantado, aunque esto no signifique que los productos sean extraordinarios en términos de factura o historia, dado el nivel formativo del alumnado. En este punto, lo más resaltante es que cada quien ve su propio avance y al final del proceso sabe los aspectos en los que debe crecer. Asimismo, la experiencia resulta enriquecedora para el personal docente, en asuntos que exceden las conjeturas iniciales, al diseñar la estrategia. En tal sentido, se puede hablar de un camino de descubrimiento en el que enseña y en el que es enseñado.

\section{Seguridad creativa}

En este aspecto se logra alcanzar solo el $65 \%$ del alumnado, debido a que no todas las personas participantes manifiestan la voluntad de comentar sus impresiones sobre las dificultades encontradas en el proyecto, por timidez o por autoconfianza. No obstante, los resultados arrojados por el análisis de los que comentaron su proceso permiten evidenciar los logros y aprendizajes individuales que se producen entre el estudiantado, pese a que en algunos de los equipos no consiguen superar los retos que le proponían sus cineminutos.

El relato audiovisual es una obra colectiva e interdisciplinar, en la medida en que se crea con la intervención de profesionales en diversas áreas, de modo que es frecuente que, durante su aprendizaje, el alumnado deba asumir cargos de producción que no termina de entender. Así, la mayoría de las conquistas particulares se refieren al dominio básico técnico-narrativo, necesario para los roles desempeñados en sus proyectos, especialmente, en el terreno de la edición, la dirección de arte y la dirección de fotografía, mientras que se aprecia cierta dificultad en los que asumen los cargos de dirección y producción, algo que no es de extrañar por la responsabilidad que suponen estos roles y por el nivel formativo de las personas participantes.

Por otra parte, se puede decir que en todos los casos en lo que se consigue hacer el seguimiento y en los que se resuelve el reto específico, se aprecia un cambio de actitud ante la actividad creativa, que se manifiesta en expresiones que pasan de una creencia de no ser capaz 
http://doi.org/10.15359/ree.26-1.8

de llegar a las soluciones, a otras que revelan entusiasmo y disposición al trabajo durante el proceso y, al final de este, satisfacción. Asimismo, en estos casos, todas las personas preguntadas al respecto tienen una consciencia clara de lo que aprendieron en la consecución del desafío, a un punto en el que no se desaniman si en su equipo no se logra la solución del problema central del cortometraje. Y, en las personas participantes que no logran el desafío individual, se aprecia que identifican claramente sus fallos. Este resultado se menciona porque, aunque no se reconoce como seguridad creativa, establece un punto de partida para futuros logros y es una manifestación reflexiva de autoconocimiento.

\section{Resolución de problemas}

En este apartado, la superación, por parte de los equipos, del principal desafío que les plantea el cineminuto muestra un número mayoritario de logro: de treinta equipos, veintiséis logran plenamente sus objetivos, mientras que cuatro no lo consiguen y uno lo hace de manera parcial. Así, se comprueba que, en todos los casos en los que se consiguen las metas, los documentos creativos evidencian una investigación básica sobre el género, que suscita reflexiones sobre el trabajo de este en sus propuestas. De igual forma, sus documentos de evaluación muestran criterios basados en la reflexión y conocimiento, si bien aún les falta consolidar competencias para realizar el trabajo con mayor solidez.

Al contrario de estos resultados, los cuatro equipos que no logran superar el desafío central del proyecto evidencian la carencia de investigación o reflexión sobre el proceso. El único equipo que se desmarca de estos resultados, logra hacer un buen cortometraje, pero no plasman sus reflexiones o investigación en el documento final, con lo que no se tiene en cuenta para los resultados de este análisis.

En cuanto a los criterios para la toma de decisiones, se hace patente cómo estos resultan efectivos para la solución de los desafíos de cada equipo. Asimismo, se revela cómo los contextos de autoevaluación y coevaluación permiten al alumnado reconocer su impronta en el fomento de la creatividad de sus pares y definir cómo quiere contar su historia, lo que le lleva a comprender que no todas las formas de hacerlo son válidas. Algo que es claro en sus explicaciones sobre si hará caso a todas las recomendaciones de los grupos focales y por qué.

Por otra parte, se observa en las personas participantes que, a medida que avanza el semestre, muestran mayor autonomía. Al comienzo del proceso, algunos no son capaces de proponer soluciones sencillas como solicitar una asesoría a profesores especialistas en el área que se les ha asignado en el equipo; otros, ni siquiera preguntan a sus compañeros o compañeras de semestres más avanzados para resolver problemas sencillos referentes a la técnica o al lenguaje audiovisual. Al final, la mayoría del alumnado no solo toma este tipo de medidas, sino que demuestran proactividad; por ejemplo, a la hora de organizar sus grupos focales y asegurarse de la comprensión de la historia por parte de la audiencia, planteando interrogantes de diferentes 
http://doi.org/10.15359/ree.26-1.8

http://www.una.ac.cr/educare

educare@una.ac.cr

maneras o grabando sus reacciones emocionales durante el visionado del producto. De igual modo, el alumnado encuentra soluciones prácticas a la carencia de algún equipo técnico, la optimización del presupuesto o la adaptación del espacio a la historia, como resultado del análisis iterativo del propio trabajo.

\section{Conocimiento del género}

Al contrastar el punto de partida de cada proyecto (documentos + prototipos) con su correspondiente entrega final (documento + cineminuto) se aprecian mejoras sustanciales en los trabajos, respecto al tratamiento genérico de la historia, mostrando cambios que hacen más clara la comunicación del relato. En consecuencia, el manejo del lenguaje audiovisual y las reflexiones técnico narrativas de sus trabajos evidencian la adquisición de nuevos conocimientos y consolidación de competencias en los miembros de los equipos, en cuestiones básicas como iluminar escenas nocturnas y tenebrosas, conseguir la tensión dramática, el empleo de los elementos de la ambientación. Aspectos que, a su vez, se concretan en un mayor entendimiento de las convenciones iconológicas y discursivas del género. Aunque cabe señalar la dificultad mostrada por el estudiantado a la hora de abordar historias de drama, frente a aquellas que pertenecen a géneros más icónicos como terror o thriller, motivo por el que se abandona trabajos de este género en la cohorte 2019-2.

En cuanto a los conocimientos teóricos y técnicos que dan base a las decisiones creativas del estudiantado, se puede decir que el desarrollo de la estrategia aplicada influye notablemente en la forma en que el alumnado entiende su trabajo en los grupos de producción con respecto a la historia, gracias a la evaluación de prototipos en cada fase y a la definición de los problemas a solucionar en cada paso. De ahí que las exposiciones en clase sobre la creación del corto reflejen un proceso reflexivo sobre la creación audiovisual.

En 2019-2, de doce equipos, once consiguen superar los desafíos de su historia; en 20191 de once cineminutos expuestos solo uno no alcanza a mejorar su prototipo, y de los diez proyectos realizados en 2018-2, solo tres no superan el nivel previo de su primer rodaje. La causa esencial en los casos que no tienen tan buenos resultados descansa en dificultades a la hora de trabajar en equipo y a la ligereza en el compromiso del alumnado con el proceso, especialmente, en la fase de empatía (investigación) y en la de definición durante la última etapa de este mismo, según lo expresado por los grupos en asesorías y parciales, y según se deduce de sus propios trabajos.

\section{Otros hallazgos}

Qué duda cabe de que una de las mejores formas de aprender es enseñar. En este caso, la aplicación de los pasos de HCD y DT por parte del estudiantado supone una fuente continua de aprendizaje para el profesorado, sobre el funcionamiento del género y sobre las formas en 
http://doi.org/10.15359/ree.26-1.8

la que puede hacer mejor su trabajo como docente. La versatilidad de la metodología facilita el diseño de un sistema en el que es relativamente sencillo hacer un seguimiento personal y grupal al avance de sus estudiantes, permitiendo atender asuntos inesperados, particulares y detectar cuestiones en las que puede afinar el proceso. En este sentido, el trabajo del estudiantado incide en el ajuste de rúbricas e instructivos y se convierten en modelos para la consulta de semestres posteriores. Por tanto, esta dinámica contribuye al avance de la clase y al fomento de la empatía en el preceptor o preceptora hacia su alumnado.

Por otra parte, la posibilidad de que todos los sujetos involucrados sean testigos de los progresos de cada integrante resulta beneficioso para el ambiente de la clase. Algo que se hace patente en la satisfacción de algunas personas, cuyos cortos no tienen altas notas, pero en lo personal consiguen superar algún reto de producción, y son reconocidas con el aplauso genuino de sus pares y de su docente en la muestra final. Y es que cabe subrayar, la buena acogida de parte del estudiantado a varias de las técnicas empleadas para la evaluación del prototipo, especialmente, la realización de grupos focales con audiencia ajena al audiovisual (ver Figura 1). Incluso, algunas personas participantes emplean esta práctica en trabajos de semestres posteriores a esta experiencia, con lo que se aprecia su aprendizaje a la hora de abordar cuestiones creativas en su trabajo audiovisual.

Figura 1: Comentario de estudiante en examen

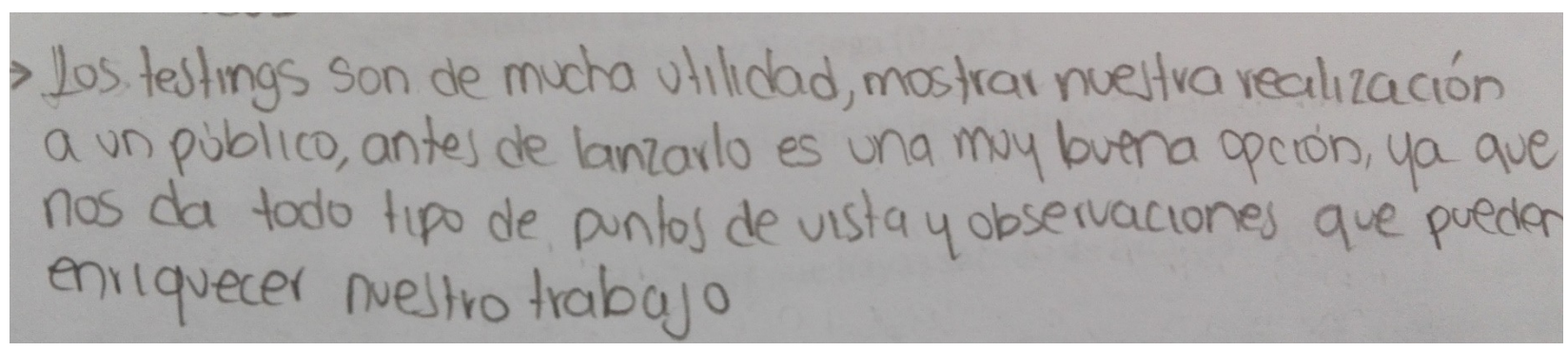

Nota: Imagen propia de esta investigación.

Otro de los resultados que se encontró en el análisis se refiere al trabajo en equipo, un factor crítico de la experiencia al ser la causa de la falta de logro en los grupos que no superaron los retos de su proyecto, lo que suscita frustración en ellos. No en vano, se percibe en sus comentarios cierta desazón por desperdiciar la oportunidad de mostrar resultados cercanos a sus expectativas, así como el reconocimiento de cierta incapacidad para la resolución de conflictos con sus compañeros y compañeras. Aun así, el alumnado que vivió esta situación refiere, en sus exámenes, haber comprendido la importancia de mantener presente el objetivo final del proyecto, que es la historia, y de cuidar la relación con sus pares de equipo como se aprecia en la Figura 2. 
http://doi.org/10.15359/ree.26-1.8

http://www.una.ac.cr/educare

educare@una.ac.cr

Figura 2: Comentario examen parcial de dos estudiantes
1. $\epsilon$ equipo trabaja en función de la tistonoa: gran aporte de Ja clase, los departamentos responden más que a Una persona a una Instoria

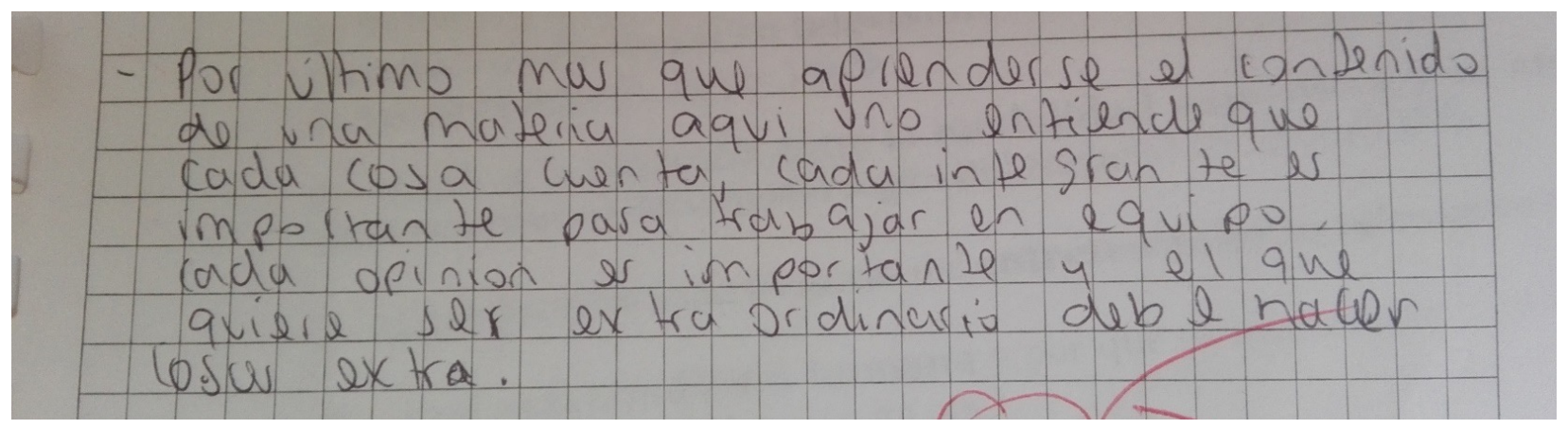

Nota: Imagen propia.

A pesar de lo anterior, se hace patente que las dificultades en este terreno deben ser tenidas en cuenta a la hora de diseñar y aplicar la estrategia, pues más allá del producto final, algunos grupos de jóvenes del curso no resuelven realmente sus diferencias y se ven incapaces de hacerlo, muchas veces por inmadurez o por la carencia de herramientas para afrontar este tipo de situaciones. En consecuencia, parece deseable abordar competencias relacionadas con la dimensión emocional del alumnado, temas como el liderazgo, la escucha atenta y la colaboración, dada su importancia para la creatividad audiovisual, pero ya no solo desde la clase, sino también desde el currículo.

\section{Conclusiones}

Tras los resultados de la sistematización y aplicación de los principios de HCD en el aula, es posible contestar el planteamiento inicial de este trabajo, afirmando que la principal bondad de esta perspectiva metodológica al audiovisual consiste en brindar una forma de exploración creativa en el aprendizaje de esta disciplina, que permite al estudiantado transitar una senda de autodescubrimiento, gracias a un ambiente positivo yoptimista de autoevaluación, coevaluación y heteroevaluación en las fases de prototipado, evaluación e ideación, en los que el alumnado debe atender otros puntos de vista, abrirse a ellos y afianzar o modificar criterios. En tal sentido, 
http://doi.org/10.15359/ree.26-1.8

HCD representa un escenario idóneo para el desarrollo de competencias relacionadas con la cocreación y el reconocimiento de la audiencia, rasgos esenciales en audiovisual.

Los beneficios que se han encontrado en las personas participantes de este caso de estudio se confirman en una mayor compresión de las dinámicas creativas audiovisuales, del rol que se espera de ellos. El fortalecimiento de su seguridad creativa se convierte en pieza clave del ambiente creativo y, sobre todo, de la actitud del estudiantado hacia su aprendizaje.

Por otra parte, se comprueba que HCD puede contribuir a la enseñanza del audiovisual, gracias al ejercicio constante del pensamiento crítico, que es fundamental para fomentar la resolución de problemas, la creatividad y el espíritu de indagación en la búsqueda de soluciones adecuadas a la variedad de desafíos técnicos y narrativos que puede suponer una producción audiovisual, gracias a las fases de prototipado, evaluación y definición que el estudiantado debe recorrer, materializando su forma de idear soluciones, de modo que para el profesorado se convierte en una manera concreta y clara de contribuir con la mejora de las propuestas del alumnado.

No cabe duda de que la seguridad creativa, que lleva a una actitud abierta y confiada en el proceso a la hora de resolver problemas está íntimamente ligada al conocimiento. En este sentido, todas las fases del HCD confrontan al alumnado con la necesidad de saber más para que su manera de abordar los problemas creativos y sus propuestas de solución sean mucho más efectivas, y se acerquen o cumplan sus expectativas respecto del producto final. Por tanto, la práctica constante e intencionada que provee el HCD contribuye a la consolidación de los conocimientos adquiridos por el estudiantado, tanto en clase como en fases de empatía, por lo que se constituye en un recurso nada despreciable para el personal docente en el terreno del audiovisual.

Los resultados obtenidos también indican que es preciso seguir probando HCD en otros temas del área, incluso ampliando la indagación a un estudiantado de semestres más avanzados, así como profundizar en cada tema abordado aquí o en asuntos curriculares de la comunicación audiovisl.

\section{Declaración de Material complementario}

Este artículo tiene disponible, como material complementario:

-La versión preprint del artículo en https://doi.org/10.5281/zenodo.4891022

\section{Referencias}

Bandura, A. (1997). Self-efficacy: The Exercise of control. W.H. Freeman.

Boy, G. A. (27 de agosto, 2013). From STEM to STEAM: Toward a human centered education. $31^{\text {st }}$ European Conference on Cognitive Ergonomics. Article 3, 1-7. https://doi. org/10.1145/2501907.2501934 
http://doi.org/10.15359/ree.26-1.8

http://www.una.ac.cr/educare

educare@una.ac.cr

Brenner, W. y Uebermickel, F. (Eds.). (2016). Design thinking for innovation, Research and practice. Springer. https://doi.org/10.1007/978-3-319-26100-3

Camillus, J. C. (2008). Strategy as a wicked problem. Harvard Business Review, 86(5), 98-101. https://goo.gl/PA5aQU

Continuum. (2012). How design thinking can help save movie theaters. Co.Disign. https://goo. $\mathrm{gl} / \mathrm{HdT} 1 \mathrm{AL}$

D'Sousa, N. (Presentadora). (9 de enero, 2016). A design thinking approach to film making [Podcast de audio]. Founding Fuel. https://goo.gl/wuYP1b

Forero Serna, A. (2018). Improvement of narrative competencies through Design Thinking in teaching of audiovisual script for undergraduate students. 25 International Conference on Learning. Universidad de Atenas.

Humnabadkar, R. (Directora). (2015), For Here or To Go? [Película]. Many Cups od Chai.

Hurbis-Cherrier, M. (2018). Voice \& vision, a creative approach to narrative filmmaking. Routledge. https://doi.org/10.4324/9781315815893

IDEO.org. (2015). The field guide to human-centered design. Autor. http://www.designkit.org/ resources $/ 1$

Jobst, B., Köppen, E., Lindberg, T., Moritz, J., Rhinow, H. y Meinel, C. (2012). The faith-factor in design thinking: Creative confidence through education at the design thinking schools potsdam and stanford? En H. Plattner, C. Meinel y L. Leifer (Eds.), Design thinking research. Measuring performance in context (pp. 35-46). Springer. https://doi.org/10.1007/978-3642-31991-4_3

Kelley, T. y Kelley D. (2013). Creative confidence, unleashing the creative potential whiting us all. Crown Business.

Kramer, J., Agogino, A. M. y Roschuni, C. (21 de ocrubre-2 de noviembre, 2016). Characterizing competencies for human-centered design. En International Design Engineering Technical Conferences \& Computers and Information in Engineering (Vol. 7: 28 ${ }^{\text {th }}$ International Conference on Design Theory and Methodology, pp. 1-12). ASME. https://goo.gl/C1 La92

Krippendorff, K. (2006). The semantic turn, a new fundation for design. CRC Press. https://doi. org/10.4324/9780203299951

Leverenz, C. S. (2014). Design thinking and the wicked problem of teaching writing. Computer and Composition, 33, 1-12. https://doi.org/10.1016/j.compcom.2014.07.001

Lugmayr A., Stockleben, B., Zou, Y., Anzenhofer, S. y Jalonen, M. (2014). Applying "design thinking" in the context of media management education. Multimedia Tools and Applications, 71(1), 119-157. https://doi.org/10.1007/s11042-013-1361-8 
http://doi.org/10.15359/ree.26-1.8

http://www.una.ac.cr/educare educare@una.ac.cr

Mackendrick, A. (2004). On filmmaking. An introduction to the craft of the director. Farrar, Straus and Giroux. https://doi.org/10.5040/9780571343713

Majithia, R. y Burman, S. (2015). Design education - evolution from markers to change makers. Conference DesignEd. Asia, Hong Kong. https://www.designedasia.com/2015/Full Papers/2015/C6 Design\%20Education\%20Evolution.pdf

Millard, K. (2014). Screenwriting in a digital era. Palgrave Macmillan. https://doi. org/10.1057/9781137319104

Pérez Guerrero, A. M. y Reyes López, C. A. (2016). Sigla, a case study on how prototypes can be used to create audio visual content and foster talent in teams formed by teachers and undergraduate students. 9th screenwriting research network conference: Screenwriting between artistic freedom and norms. Universidad de Leeds Becket.

Rodríguez Martín, J. D. (10-11 de mayo, 2018). Distribución por festivales de productos audiovisuales universitarios a partir del HCD. 1 encuentro de semilleros en investigación creación audiovisual. Universidad de La Sabana.

Rodríguez Rojas, F. y Mosquera, C. (7-11 de mayo, 2018). Laboratorio "Relatos del patrimonio en clave digital". Análisis desde la producción digital y la colaboratividad. En Londoño, F. (director), 17 festival internacional de la imagen. Nuevas expediciones. Universidad de Caldas, Manizales, Colombia.

Steen, M. (2012). Human-centered design as a fragile encounter. Design Issues, 28(1), 72-80. https://doi.org/10.1162/DESI a 00125

Steinbeck, R. (2011). El "design thinking" como estrategia de creatividad en la distancia., Comunicar, 19(37), 27-35. http://dx.doi.org/10.3916/C37-2011-02-02

Tschimmel, K., Loyens, D., Soares, J. y Oraviita, T. (2017). D-think toolkit. ESAD, ERASMUS+ KA2 Strategic Partnerships. https://goo.gl/um5CKh

Tschimmel, K., Santos, J., Loyens, D., Jacinto, A., Monteiro, R. y Valença, M. (2015). Research report $D$-Think, Design thinking applied in education and training- ESAD, ERASMUS+ KA2 Strategic Partnerships. https://www.academia.edu/25633323/Research Report D Think Design Thinking Applied to Education and_Training

Universidad de La Sabana, Facultad de Comunicación. (2018). Programa de géneros y formatos audiovisuales [Carrera Comunicación Audiovisual y Multimedios].

van der Bijl-Brower, M. y Dorst, K. (2017). Advancing the strategic impact of human-centred design. Design Studies, 53, 1-23. http://dx.doi.org/10.1016/j.destud.2017.06.003 